(1)

CrossMark

\title{
Moving forward from drug-centred to patient-centred research
}

\author{
A white paper initiated by EORTC and developed together with the BioMed \\ Alliance members
}

\author{
Denis Lacombe ${ }^{1}$, Colm O'Morain ${ }^{2}$, Barbara Casadei ${ }^{3}$, Kate Hill $\mathbb{1}^{4}$, \\ Elsa Mateus ${ }^{5}$, Rik Lories ${ }^{6}$ and Guy Brusselle ${ }^{7}$
}

Affiliations: ${ }^{1}$ Director General, European Organisation for Research and Treatment of Cancer. ${ }^{2}$ Past President, Alliance for Biomedical Research in Europe and United European Gastroenterology. ${ }^{3}$ President, European Society of Cardiology. ${ }^{4}$ Chair of the ELF Patient Advisory Committee, European Lung Foundation. ${ }^{5}$ EULAR/PARE representative, Patient Research Partner. ${ }^{6}$ EULAR representative to BioMed Alliance, European League Against Rheumatism. ${ }^{7}$ Chair of the ERS Science Council, European Respiratory Society.

Correspondence: Denis Lacombe, Director General, European Organisation for Research and Treatment of Cancer, Avenue Mounier 83/11, Brussels, 1200, Belgium. E-mail: denis.lacombedeortc.org

@ERSpublications

This paper discusses how to restructure the process of clinical research to maximise the potential of precision medicine http://ow.ly/1ZCc30nuw2a

Cite this article as: Lacombe D, O'Morain C, Casadei B, et al. Moving forward from drug-centred to patient-centred research. Eur Respir J 2019; 53: 1801870 [https://doi.org/10.1183/13993003.01870-2018].

\section{Introduction}

Maximising the potential of precision medicine for patients and healthcare services is a major societal challenge. It requires a holistic approach to the development of therapeutic strategies and a re-thinking of the entire process, including the role of the respective stakeholders and the way they interact, from the early steps of drug development to access in real life. First, the new technologies that inform us about the biology of the disease and enable better treatments plead for a reversal of the "protocols search patients" approach, to "patients searching (the best possible) treatments and protocols". Second, new drugs reaching the market is not an end but a start. Information that is critical for the integration of new treatments in daily practice needs to be collected and analysed to optimise the use of resources and maximise patient benefits. Optimal dose, sequence, combination and duration of treatments as well as cut-off values of biomarkers and their clinical utility all represent crucial pieces of information not only for patients and doctors, but also for healthcare systems facing complex decisions on reimbursement and access. The gap that currently exists between market approval and real-life clinical practice, and that is not addressed by the commercial sector, requires a new infrastructure for applied clinical research which needs to be fully integrated into the cycle of drug development, market approval and clinical application. This process needs to be re-engineered in a such a way that it truly serves the needs of patients and generates the data needed to inform clinical practice.

\section{Rationale for change}

An unprecedented speed in the growth of knowledge, combined with the availability of large prospective cohorts and the emergence of new technologies, is enabling more effective validation of therapeutic targets and pharmacogenomics [1]. Over the past few years, empirical drug development in a single clinically and pathologically defined disease has been challenged by the identification of molecularly or genetically 
defined subsets of patients amenable to targeted treatments. In the cancer field, "histology agnostic trials" testing new drugs aimed at molecular targets rather than at the same tumour are already part of the clinical research landscape, leading to registration of new agents based on molecular features $[2,3]$.

Taking the full regulatory and scientific environment into account is critical. The first necessity is to develop a different framework, where more refined patient stratification and individualised effective care, rather than drug development, are at the centre of the process [4]. Furthermore, this framework should encompass and support the structured involvement of patients, as experts, research partners and active stakeholders, providing their experiential knowledge $[5,6]$.

Patients must remain the focus throughout the discovery process, whilst the regulatory framework for testing new interventions in a robust and meaningful way is revisited. Moreover, since the extent of patient involvement in regulatory issues varies considerably between countries and regions in Europe, the development of frameworks by the national competent authorities must also envisage an active participation and close interaction with patients, ensuring the input of their real-life experiences of diseases and their management into the process [7].

Patients' needs are multiple; most commonly they do not depend on a single intervention and are likely to change with the natural history of the disease. Therefore, the concept that therapeutic interventions are developed with the sole purpose of market access, not anticipating any questions beyond registration/ market authorisation, needs to be revisited. This new approach should cover not only pharmaceuticals, but also other modalities, such as surgery, diagnostics and screening.

Similarly, questions surrounding the sequence and combinations of multiple therapeutic interventions need to be addressed to rationalise implementation of agents and better understand their value in the therapeutic armamentarium. Typically, all these questions are addressed by the non-commercial sector through applied clinical research. After market authorisation, long-term safety and effectiveness in broader patient populations need to be monitored [8]. Regulatory agencies are already beginning to require such information [9].

Taken together, these considerations highlight the need for a profound transformation in the development cycle of any therapeutic intervention and for a departure from the comfort zone within which many stakeholders now operate [10].

The current regulatory framework has resulted in a dramatic increase in the cost of conducting randomised clinical trials (RCTs), without increasing patient safety [11]. Investigator-led trials are increasingly difficult to conduct and the number of new interventions and optimised therapeutic strategies that can be tested has decreased dramatically. This development represents a major threat to our healthcare system. At present, research protocols are written to fit existing rules for drug development rather than in the interest of finding the best solution for stakeholders. This must change and change soon. There is robust evidence supporting the implementation of streamlined applied clinical research, but still much uncertainty as to whether it will be embraced by all stakeholders. Thus, it is vital that regulatory authorities and also health technology assessment (HTA) bodies engage with the non-commercial sector over the disruptive knowledge that accompanies the advent of precision medicine.

The following principles underpin the strategy proposed by the BioMed Alliance:

- Patients have the right to participate at each strategic level in health research. This includes full participation at all decision levels and stages of clinical trials, from research priorities and protocol design, to treatment development, project reviewing, and dissemination and implementation of results [12, 13].

- Patients have the right to benefit from the latest scientific discoveries and to be treated according to the highest level of clinical evidence. Technologies allowing the stratification of patients according to biological/genetic features should lead us to change our models: from "drug protocols looking for patients" to (fully biologically/genetically characterised) "patients looking for matching protocols" [14]. Thus, truly placing patients at the centre of the research and development $(\mathrm{R} \& \mathrm{D})$ process, where they should be partners from its inception.

- Researchers and clinicians should support patients by providing timely and relevant information about trials of new drugs and emerging technologies in a format that is accessible to patients and promotes meaningful involvement. Patients should also be better informed about the value of clinical trials and more involved in trial design and the choice of primary outcomes.

- New solutions are needed for optimal benchmarking of emerging technologies across and within a class of agents. The concept of "one intervention, one target, one protocol" is no longer the way forward.

- Adaptive designs for clinical trials are an opportunity to increase the efficiency of RCTs, since it enhances the likelihood of finding a true benefit, if one exists, of the treatment(s) being tested [15]. In exploratory RCTs, adaptive designs facilitate finding effective and safe doses of drug(s), whereas in 
confirmatory RCTs, the adaptive design refers to prospectively planned changes to the future course of an ongoing trial based upon analyses of accumulating data from the trial itself [15].

- Key questions anticipating real-life implementation of new interventions need to be addressed early on, i.e. combinations, duration of treatments, long-term safety in patients with multimorbidity and/or polypharmacy $[16,17]$. These types of questions are crucial not only for patients, but also for HTA bodies and payers [18]. Hence, the importance of the involvement of patients, as partners in the research team and in HTA-related procedures [19].

- Long-term toxicity monitoring of mechanism-based therapies needs to extend beyond registration into real life for a prolonged period. There should be a continuum between drug development, regulatory assessment, clinical research and applied clinical research. Patients should also be educated for monitoring and reporting the effects of the therapeutic intervention [20].

- Trial end-points should take into consideration outcomes that reflect the needs and priorities of patients (not just of regulators) [21]. Therefore, patients must be involved at each strategic level in health research.

\section{Infrastructural gaps}

There is much room for improvement in the process of bringing the latest science to patients, while taking into account their priorities for treatment and their preferences for quality of life versus longevity. Too often, regulatory agencies, governments and funding agencies do not encourage the integration of research into care, and vice versa. Similarly, the pharmaceutical drug development process remains protected during the competitive phase, placing drug development priorities before public health issues when the continuum of care would require early consideration of these issues, a broader view and a more comprehensive approach. While continuing to preserve the interest and needs of all stakeholders, a substantial waste of knowledge and resources must be avoided.

The following principles underpin the strategy proposed by the BioMed Alliance:

- There needs to be an integrated pan-European infrastructure to support the use of patient data for health research. Such a system circumvents the expense of active long-term follow up (and thus, allows adequate assessment of safety and cost-effectiveness of interventions) and provides information that is accessible for independent assessment by health authorities. European-wide clinical population-based registries, encompassing information on clinical data, biological and imaging data, biomarker test results, data on all therapies received and outcomes (e.g. via electronic patient records), are critical for the affordable implementation and validation of state-of-the-art precision medicine. Controlling the quality and accuracy of the data is essential and infers that minimal quality control requirements for building these databases and registries will need to be developed and implemented. Patients should also be empowered into (individual) data ownership and protection, as much as on the relevance of data.

- Traditionally, HTA bodies and payers base their decisions on drug development research, which remains relatively artificial (often studying highly selected patient populations and providing only short-term safety and efficacy data). We argue that infrastructures such as a clinical population-based registry would allow, when patients have provided informed consent, their recruitment into clinical trials directly from clinical services, better reflecting real life and providing long-term safety data and comparative effectiveness data [22].

- Observational and interventional studies performed within this pan-European infrastructure should have adequate power and sample sizes according to the specific research question to be addressed. Whereas clinical research often needs large samples, capturing more high-dimensional biological information about individual patients might decrease significantly the required sample sizes for distinguishing phenotypes [23].

\section{Conceptual changes are needed}

Drug development protocols are usually written with the aim of bringing a new agent to the market for a very specific clinical situation. They do not test the optimal integration of a new drug into existing therapeutic strategies, such as how to combine treatment, in which sequence, and for how long. While short-term regulatory trials are needed to demonstrate therapeutic benefit, they may not address real-life issues (such as those arising from disease progression or even from the social determinants of health) and may fail to capture rare or delayed safety outcomes arising from drug exposure.

There is, thus, a missing link between regulatory trials and healthcare systems. Implementing applied clinical research to address this missing link must be considered [24]. An example can be the advent of immuno-oncology, as no information is available on the optimal duration of treatment with so called 
"check-point inhibitors", despite their costs and potential long-term side-effects. Smart application of (single or composite) biomarkers for patient selection and patient monitoring will be critical in achieving this goal. Moreover, applied clinical research will be key in determining the optimal cut-off values of appropriate biomarkers in diverse disease entities and their heterogeneous clinical phenotypes. These solutions will help place the continuum of drug development for optimal patient access at the forefront of clinical trials and will enable applied clinical research in real life. Tailor-made solutions could open doors to integration with population-based registries, reducing costs, improving the patient journey and allowing investigator-initiated and patient-centred assessment of relevant therapeutic interventions.

\section{Defined objectives}

Two major issues that need to be addressed at European level in order to achieve a successful transition to precision medicine are:

- The establishment of Europe-wide clinical population-based registries, which will provide the infrastructure and data quality control for patient-centred, affordable, real-life testing of new and repositioned treatment strategies.

- Optimising treatment in real life based on robust evidence, taking into account key patient-centred questions for real-life implementation such as optimal dose, duration, sequence, combination, and quality of life.

For any transition to succeed, a balance must be found between the interests and needs of all stakeholders:

- Patient triage (molecular/genetic screening) and trial access: academia in partnership with pharma, biotech, diagnostics, etc.

- Drug development: industry in partnership with researchers, medical societies, patient organisations and relevant agencies.

- Therapeutic optimisation led by the non-commercial sector: academia in partnership with HTA and payers, with the involvement of patients' representatives.

- Real-life implementation and long-term monitoring of treatments led by the non-commercial sector: academia in partnership with registries, engaged patients' organisations, HTA, patients and payers.

A major transformation of clinical research, building on the strengths and complementarity of stakeholders working alongside new business models, must be tackled in order to make the above possible, notably developing strategies for chronic diseases.

\section{A vision for the future}

We strongly advocate that both $\mathrm{R} \& \mathrm{D}$ in healthcare and its regulatory framework need to involve all stakeholders.

Widespread availability of imaging, molecular, genetic and biochemical biomarkers derived from prospective cohorts, and patients' medical records is necessary to refine patient selection to clinical trials. A European-wide infrastructure that allows patient identification and affordable long-term follow up, for instance via their electronic medical records, is essential to the practice of "intelligent" and sustainable healthcare.

The model we propose would be patient-centred (as patients would be actively involved and gain access to the most suitable treatment) and would optimise the understanding of patterns of recurrence or failure, informing investment in new R\&D strategies.

It is critical that patient information for health research be obtained from databases that are curated and constantly updated. The existence of such infrastructure would allow not only optimal selection of patients in clinical trials, but also enable long-term follow-up of all patients as well as benchmarking of clinical research in real life with no loss to follow-up [25].

It is urgent that European bodies that have the capacity to stimulate such changes get their acts together if we want to make precision medicine a viable option, rather than a chance happening that generates false hope for patients and the scientific community.

We propose to re-discuss the architecture of the process of clinical research and explore the design and maintenance of clinical outcome-focused systems, which have anticipated real-life questions early on in their development, through the involvement of patients. We will work in partnership with patients and the public to co-produce solutions that will enable the potential of the vast amount of routinely collected data to be utilised [26]. Such a change in architecture is needed, not only for maximising the potential of scientific advances for individual patients, but also for bringing economic benefit to healthcare services through the ability to target new and established treatment to those who are certain to benefit from it. 
To change the current architecture of the process of clinical research, we urge European Institutions to work closely with member states and to implement the following policy actions:

- Missions are a major new element in Horizon Europe, the flagship programme of research and innovation at the European Union (EU) level. Bringing together all EU patient data in such a single pan-European infrastructure could be a strong health research mission within Horizon Europe. The collection of patient data, first on a national scale (where this is not already being done) and subsequently EU-wide, will be a crucial step towards eradicating many of the diseases that place such a heavy burden on EU societies. Technologically feasible, the creation of a European repository for patient data will require significant funding and the overcoming of legal, ethical, political and educational challenges. To do this would mean that trials would be not only patient-centred, as patients would gain access to the most suitable treatment, but that they would also optimise the understanding of patterns of recurrence or failure, informing investment in new R\&D strategies. And, of course, it would enable the long-term follow-up of all patients.

- The next framework programme, Horizon Europe, should include health research calls aimed at supporting applied clinical research and therapeutic optimisation studies (e.g. pragmatic trials comparing the effectiveness of different treatment options in real life).

- The EU, in partnership with its member states, should establish therapeutic optimisation research as an official and mandatory step in the treatment access path to market, while ensuring this does not lead to further delays in patients' access to innovative treatments.

- National legislation should include provisions allowing for publicly funded international research to address collective therapeutic challenges. Member states should agree on a framework for joint optimisation research whenever there is need for an international approach.

Acknowledgements: The authors thank the following people for their contribution to the article: Ioana Agache (President, EAACI), Peter Van Den Bergh (Member of European Affairs Subcommittee, EAN), Kristoff Muylle (President, EANM), Lale Tokgözoglu (President, EAS), Alberico Catapano (Past President, EAS), Anders Bjartell (Chairman of the EAU Research Foundation, EAU), Javier Gisbert (Clinical Research Committee, ECCO-IBD), Guy Joos (Past President, ERS), Roy Farquharson (Chairman, ESHRE), Tony Lahoutte (President, ESMI), Marc Benninga (Member and Treasurer of Gastroenterology Committee, ESPGHAN), Emmanuel Fragkoulis (Chair of the Science and Society Committee, FEBS), Isabel Varela Nieto (Member of the Science and Society Committee, FEBS), Frank Rümmele (Member of Research Board, UEG).

Endorsed by the Board of Directors of Biomedical Alliance in Europe (BioMed Alliance; www.biomedeurope.org) and the Academic Clinical Trials Task Force which is composed of the following BioMed Alliance member societies: European Academy of Allergy and Clinical Immunology (EAACI; www.eaaci.org), European Academy of Neurology (EAN; www.ean.org), European Association for Study of the Liver (EASL; www.easl.eu), European Association for the Study of Obesity (EASO; www.easo.org), European Association of Nuclear Medicine (EANM; www.eanm.org), European Association of Urology (EAU; www.uroweb.org), European Atherosclerosis Society (EAS; www.eas-society. org), European CanCer Organisation (ECCO; www.ecco-org.eu), European Crohn's and Colitis Organisation (ECCO-IBD; www.ecco-ibd.eu), European Hematology Association (EHA; www.ehaweb.org), European League Against Rheumatism (EULAR; www.eular.org), European Organisation for Research and Treatment of Cancer (EORTC; www. eortc.org), European Respiratory Society (ERS; www.ersnet.org), European Society for Molecular Imaging (ESMI; www. e-smi.eu), European Society for Paediatric Gastroenterology Hepatology and Nutrition (ESPGHAN; www.espghan.org), European Society of Anaesthesiology (ESA; www.esahq.org), European Society of Cardiology (ESC; www.escardio.org), European Society of Human Reproduction and Embryology (ESHRE; www.eshre.eu), Federation of European Biochemical Societies (FEBS; www.febs.org), United European Gastroenterology (UEG; www.ueg.eu).

The Biomedical Alliance in Europe (the BioMed Alliance) has 29 members, all of whom are scientific and professional medical associations, which are active in all aspects of their specialties across the whole of Europe. It was created in 2010 to establish a mechanism for biomedical and clinical researchers to provide a collective voice to the European Institutions in support of medical research. The BioMed Alliance's principal goals and objectives are to promote the best interests and values of researchers and of healthcare professionals, organised in non-for-profit scientific medical associations and organisations across all medical disciplines in Europe, in those general areas where common interest is identified. The BioMed Alliance's scientific societies collaborate closely with European-wide patient organisations in the respective disease domains in order to improve patient outcomes and to strengthen patient participation in clinical research. The BioMed Alliance has advocated for an increase in funding for Horizon Europe (particularly for health research) and for the implementation of structural measures to accommodate sustainability for research programmes and to streamline activities aiming to support basic research and collaborative translational biomedical research, and to close the gap between basic research and clinical practice. The BioMed Alliance has been advocating for bottom-up initiatives in designing health research programmes, for more visibility of EU research, and for precision medicine-based patient-centred solutions in designing and conducting clinical trials.

Author contributions: All authors contributed for important intellectual content and approved the final manuscript.

Conflict of interest: D. Lacombe has nothing to disclose. C. O'Morain has nothing to disclose. B. Casadei has nothing to disclose. K. Hill has nothing to disclose. E. Mateus reports personal fees from EULAR, in form of a services agreement regarding consultancy for patient involvement in the HarmonicSS Project (this project has received funding from the European's Union Horizon 2020 Research and Innovation Programme under grant agreement number 731944 and from the Swiss State Secretariat for Education, Research and Innovation SERI under grant agreement 16.0210), outside the submitted work. R. Lories reports institutional fees for consultancy and speaker activities from Celgene, Janssen, 
Samumed, Galapagos, Novartis, Eli Lily, Abbvie, UCB and Pfizer, outside the submitted work. G. Brusselle has nothing to disclose.

\section{References}

$1 \quad$ Wang L, McLeod HL, Weinshilboum RM. Genomics and drug response. N Engl J Med 2011; 364: 1144-1153.

2 Woodcock J, LaVange LM. Master protocols to study multiple therapies, multiple diseases, or both. $N$ Engl J Med 2017; 377: 62-70.

3 André F. Developing anticancer drugs in orphan molecular entities - a paradigm under construction. $N$ Engl $J$ Med 2018; 378: 763-765.

4 Moher D, Glasziou P, Chalmers I, et al. Increasing value and reducing waste in biomedical research: who's listening? Lancet 2016; 387: 1573-1586.

5 de Wit MPT, Berlo SE, Aanerud GJ, et al. European League Against Rheumatism recommendations for the inclusion of patient representatives in scientific projects. Ann Rheum Dis 2011; 70: 722-726.

6 Geissler J, Ryll B, di Priolo SL, et al. Improving patient involvement in medicines research and development: a practical roadmap. Ther Innov Regul Sci 2017; 51: 612-619.

7 Haerry D, Landgraf C, Warner K, et al. EUPATI and patients in medicines research and development: guidance for patient involvement in regulatory processes. Front Med (Lausanne) 2018; 5: 230.

8 Roche N, Reddel HK, Agusti A, et al. Integrating real-life studies in the global therapeutic research framework. Lancet Respir Med 2013; 1: e29-e30.

9 Gassman AL, Nguyen CP, Joffe HV. FDA regulation of prescription drugs. N Engl J Med 2017; 376: 674-682.

10 Giacomi FD, Vassallo R, Yi ES, et al. Acute eosinophilic pneumonia. causes, diagnosis, and management. Am J Respir Crit Care Med 2018; 197: 728-736.

11 Reith C, Landray M, Devereaux PJ, et al. Randomized clinical trials - removing unnecessary obstacles. $N$ Engl J Med 2013; 369: 1061-1065.

12 Kirwan JR, de Wit M, Frank L, et al. Emerging guidelines for patient engagement in research. Value Health 2017; 20: 481-486.

13 Price A, Albarqouni L, Kirkpatrick J, et al. Patient and public involvement in the design of clinical trials: An overview of systematic reviews. J Eval Clin Pract 2018; 24: 240-253.

14 Rothwell PM. External validity of randomised controlled trials: "To whom do the results of this trial apply?". Lancet 2005; 365: 82-93.

15 Bhatt DL, Mehta C. Adaptive designs for clinical trials. N Engl J Med 2016; 375: 65-74.

16 Carson SS, Goss CH, Patel SR, et al. An official American Thoracic Society research statement: comparative effectiveness research in pulmonary, critical care, and sleep medicine. Am J Respir Crit Care Med 2013; 188: $1253-1261$.

17 Lieu TA, Au D, Krishnan JA, et al. Comparative effectiveness research in lung diseases and sleep disorders. Am J Respir Crit Care Med 2011; 184: 848-856.

18 Ford I, Norrie J. Pragmatic trials. N Engl J Med 2016; 375: 454-463.

19 Hunter A, Facey K, Thomas V, et al. EUPATI guidance for patient involvement in medicines research and development: health technology assessment. Front Med (Lausanne) 2018; 5: 231.

20 Inácio $\mathrm{P}$, Cavaco $\mathrm{A}$, Airaksinen $\mathrm{M}$. The value of patient reporting to the pharmacovigilance system: a systematic review. Br J Clin Pharmacol 2017; 83: 227-246.

21 Concato J. Study design and "evidence" in patient-oriented research. Am J Respir Crit Care Med 2013; 187: 1167-1172.

22 Gelijns AC, Gabriel SE. Looking beyond translation - integrating clinical research with medical practice. $N$ Engl J Med 2012; 366: 1659-1661.

23 Li C-X, Wheelock CE, Sköld CM, et al. Integration of multi-omics datasets enables molecular classification of COPD. Eur Respir J 2018; 51: 1701930.

24 Custovic A, Belgrave D, Lin L, et al. Cytokine responses to rhinovirus and development of asthma, allergic sensitization and respiratory infections during childhood. Am J Respir Crit Care Med 2018; 197: 1265-1274.

25 Keet CA, Keller JP, Peng RD. Long-term coarse particulate matter exposure is associated with asthma among children in Medicaid. Am J Respir Crit Care Med 2018; 197: 737-746.

26 Goytia CN, Kastenbaum I, Shelley D, et al. A tale of 2 constituencies: exploring patient and clinician perspectives in the age of big data. Med Care 2018; 56: Suppl. 10, S64-S69. 